\title{
Chromatic Dispersion measurement of short optical fibers by sinusoidal phase modulation of a monochromatic light
}

\author{
Aliou Ly, Gilles Bailly, Arnaud Fernandez, Olivier Llopis \\ LAAS-CNRS, Université de Toulouse, CNRS, UPS \\ 7 av. du Colonel Roche, 31031 Toulouse, France
}

aly@1aas.fr

\begin{abstract}
We report a simple method to measure the chromatic dispersion of short length optical fibers using phase modulation of a continuous-wave light. The results obtained were exploited to design a coupled optoelectronic oscillators with optimization of its intracavity dispersion.
\end{abstract}

Keywords - chromatic dispersion, optical fiber, phase modulator, microwave optics, optoelectronic oscillator

\section{INTRODUCTION}

Chromatic dispersion (CD) in single mode optical fibers is caused by two effects: the material dispersion and the waveguide dispersion of the fiber. The former is due to the wavelength dependence of the refractive index of glass. The latter is due to the fiber's guiding properties. In both cases, it is well known that chromatic dispersion has detrimental effects in high data rate optical fiber communications as well as in radio over fiber transmission systems [1]. As a result, many and well established techniques have been proposed for precise measurement of CD. Among them one can mention the phase shift based method, optical interferometry or the microwave interference effect [2].

However, it is worth noticing all those methods were implemented to measure CD of optical fibers with lengths of tens of kilometers because of the above mentioned applications. Thus, it is quite striking to see that CD measurement of short length optical fibers (hundreds of meters typically) have gained less attention whereas there are some applications in which this is necessary. This is the case for the coupled optoelectronic oscillator (COEO). Indeed, the spectral purity of the RF signal generated by this device strongly depends on its cavity dispersion [3]. A recent work aiming at measuring this dispersion has been reported [4].

Nevertheless, in a primary step of designing a COEO, it would be advantageous to precisely measure the $\mathrm{CD}$ of its optical fiber loops. In this frame, we present here the measurement of short optical fibers CD by mean of a sinusoid ally phase modulated continuous-wave (cw) light. To the best of our knowledge, only one work using this approach has been reported to date and it was applied to very long optical fibers [5].

\section{CD MEASUREMENT PRINCIPLE AND THEORY}

\section{A. CD measurement principle}

The experimental setup for CD measurement is depicted in Fig. 1. The cw laser is phase modulated by a $10 \mathrm{GHz}$ wideband phase modulator (MPZ-LN Photline). A polarization controller ensures that the incident light enters the modulator with the right polarization. The phase modulated light next travels a dispersive medium, the fiber under test (FUT) before being detected by a photodiode (Discovery DSC30S). The modulation depth $\theta_{\mathrm{m}}$ will be weak enough to assume that the laser spectrum at the output of the modulator will consist mainly in the carrier and two optical sidebands which are $180^{\circ}$ out of phase.

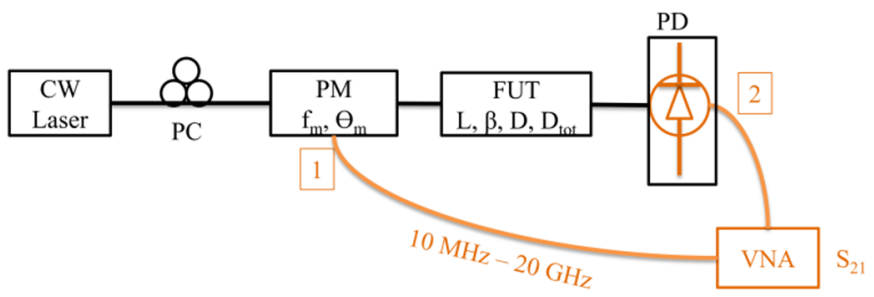

Fig. 1. Experimental setup for $\mathrm{CD}$ measurement: PC: polarization controller, PM: phase modulator, FUT: fiber under test, PD: photodiode, VNA: vector network analyzer.

The FUT is characterized by its geometrical length L, its complex propagation constant $\beta$, its CD per unit length of fiber $\mathrm{D}$ in $(\mathrm{ps} / \mathrm{nm} / \mathrm{km})$ and its accumulated CD over the length $\mathrm{L}, \mathrm{D}_{\text {tot }}$ in (ps/nm). The two optical sidebands will be affected differently by the dispersion of the FUT when propagating through it. This will cause the amplitude of the recovered modulation to vary with modulating frequency as a result of a phase to intensity modulation conversion that takes place inside the FUT. Thus, by measuring this amplitude variation one can extract the CD of the FUT. To do so, a vector network analyzer (VNA, ZVB20 Rohde\&Schwarz) is used as a RF signal generator and receiver. The RF signal frequency $f_{m}$ is swept from $10 \mathrm{MHz}$ to $20 \mathrm{GHz}$ and the gain of the $S_{21}$ parameter is measured. The next paragraph will show how the measurement of this gain can be processed to extract the CD of the FUT. 


\section{B. Theory}

To see how the phase modulated light behaves after propagating through the FUT, let's start with the usual expression for a sinusoidally phase modulated light:

$$
\begin{array}{r}
E_{\text {in }}(t)=\frac{A_{\text {in }}}{2}\left[J_{0}\left(\theta_{m}\right) e^{-i \omega_{c} t}+J_{1}\left(\theta_{m}\right) e^{-i\left(\omega_{c}+\omega_{m}\right) t}\right. \\
\left.+J_{-1}\left(\theta_{m}\right) e^{-i\left(\omega_{c}-\omega_{m}\right) t}\right]+ \text { c. c } .
\end{array}
$$

Where c.c. stands for complex conjugate to take into account that the electric field is real, $\omega_{c}$ is the carrier angular frequency and $\omega_{\mathrm{m}}$ corresponds to the angular modulation frequency. In this equation we assume weak modulation so as the phase modulated light can be expanded into Bessel functions of first kind by only keeping the order +1 and -1 . At the output of the FUT, the expression of the electric field becomes:

$$
\begin{aligned}
E_{\text {out }}(t)=\frac{A_{\text {in }}}{2}\left[J_{0}\right. & \left(\theta_{m}\right) e^{-i \omega_{c} t} \\
& +J_{1}\left(\theta_{m}\right) e^{-i\left(\omega_{c}+\omega_{m}\right) t} \\
& \left.+J_{-1}\left(\theta_{m}\right) e^{-i\left(\omega_{c}-\omega_{m}\right) t}\right] e^{i \beta L} \\
& + \text { c.c. } .
\end{aligned}
$$

The imaginary part of the complex propagation constant $\beta$ leads to attenuation of the modulated light through the fiber and its real part causes chromatic dispersion. It can be expanded in Taylor series as follows:

$$
\begin{aligned}
\beta_{r}(\omega)=\beta_{r}\left(\omega_{c}\right) & +\dot{\beta}_{r}\left(\omega_{c}\right)\left(\omega-\omega_{c}\right) \\
& +\frac{\dot{\beta}_{r}}{2}\left(\omega_{c}\right)\left(\omega-\omega_{c}\right)^{2}
\end{aligned}
$$

The third term in Eq. (3) is responsible for the distortion and the broadening of the optical signal. Indeed, it is related to CD per unit length by the following expression:

$$
D=-\frac{2 \pi c}{\lambda^{2}} \ddot{\beta}_{r}\left(\omega_{c}\right)
$$

The photocurrent produced at the output of the photodiode can then be written as:

$$
\begin{aligned}
I_{P D}(t)=\alpha \eta P_{\text {in }}[ & J_{0}\left(\theta_{m}\right) \cos \left(\omega_{c} t\right) \\
& +J_{1}\left(\theta_{m}\right) \cos \left(\left(\omega_{c}+\omega_{m}\right)\right. \\
& \left.+\varphi_{m}\right) \\
& +J_{-1}\left(\theta_{m}\right) \cos \left(\left(\omega_{c}+\omega_{m}\right) t\right. \\
& \left.\left.+\varphi_{m}\right)\right]^{2}
\end{aligned}
$$

Where $\alpha=e^{-2 \beta_{i} L}$ is the FUT attenuation which is assumed to be the same for all frequency components, $\eta$ the response of the photodiode in $\mathrm{A} / \mathrm{W}$ and $\varphi_{\mathrm{m}}$ is given by:

$$
\varphi_{m}=-\frac{\pi L D}{c} \lambda^{2} f_{m}^{2}
$$

It corresponds to the phase distortion induced by the dispersion of the FUT.

The photocurrent in Eq. (5) has a DC component and contains terms oscillating at the modulation frequency and high order harmonics. The DC current allows the measurement of the FUT attenuation by comparing its value with and without the
FUT. After expanding Eq. (5) and applying the appropriate trigonometric formula, the sum of the oscillating terms at $\omega_{\mathrm{m}}$ converted into a voltage at the VNA input is:

$$
\begin{aligned}
& V_{R F}(t) \\
& =\alpha \eta R_{L} P_{i n} J_{0}\left(\theta_{m}\right) J_{1}\left(\theta_{m}\right) \sin \left(\frac{\pi L D}{c} \lambda^{2} f_{m}^{2}\right) \sin \left(2 \pi f_{m} t\right)
\end{aligned}
$$

With $\mathrm{R}_{\mathrm{L}}$ the $50 \Omega$ load resistance of the VNA. It can be clearly seen in this equation the process of dispersion induced phase distortion conversion to amplitude modulation. The $\mathrm{S}_{21}$ gain measured at the VNA is related to the amplitude of the recovered modulation in Eq. (7) as follows:

$$
\left|S_{21}\right|=20 \log _{10}\left(\frac{V_{R F}(t)}{\sqrt{R_{L}}}\right)-P_{\text {in }}
$$

Where $\mathrm{P}_{\text {in }}$ in $(\mathrm{dBm})$ is the RF power at the phase modulator input. Thus, from the measurement of the VNA, one can determine the amplitude of the recovered modulation which evolves sinusoidally versus the square of the modulating frequency. A way to subsequently retrieve the $\mathrm{CD}$ per unit length $\mathrm{D}$ is to look at the frequency at which the sine cancels. This was done in [5], but, as stated earlier, it requires long fiber lengths for moderate modulation frequencies. In our case, we will just take advantage of the linear part of this sine function. By fitting its linear part, one can extract the total dispersion $\mathrm{D}_{\text {tot }}$ of the FUT from the obtained slope. However, since this slope depends on parameters that may fluctuate, a prior calibration is necessary before characterizing the dispersion of short optical fibers.

\section{CD MEASUREMENT OF SMF FIBERS}

The results below are all obtained under the following experimental conditions: the laser operating wavelength was set at $1552 \mathrm{~nm}$ with an output power of $13 \mathrm{dBm}$ and the RF power from the VNA was fixed at $\mathrm{P}_{\text {in }}=7 \mathrm{dBm}$.

\section{A. Calibration with a $155 \mathrm{~m}$ SMF-28 fiber}

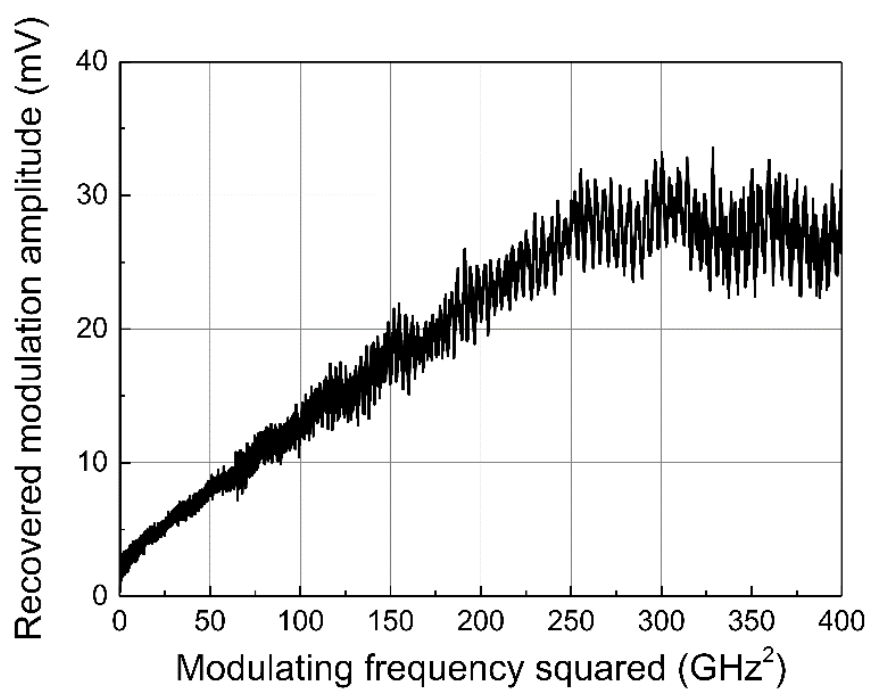

Fig. 2: Evolution of the recovered modulation amplitude for a $155 \mathrm{~m}$ fiber. 
The slope of the linear part of the amplitude of the sine function in Eq. (7) can be written as:

$$
p=\alpha \eta R_{L} P_{i n} J_{0}\left(\theta_{m}\right) J_{1}\left(\theta_{m}\right) \frac{\pi L D}{c} \lambda^{2} \equiv \alpha \kappa D_{t o t}
$$

The calibration consists then in determining the value of the constant $\kappa$. Indeed, once its value is known, the total dispersion for any FUT with an attenuation of $\alpha_{\mathrm{FUT}}$ is given by:

$$
D_{\text {tot }}=\frac{p_{\text {measured }}}{\alpha_{\text {FUT }} \kappa}
$$

To this aim, we use a $155 \mathrm{~m}$ length SMF-28 fiber as a calibration FUT with a known total dispersion of $2.64 \mathrm{ps} / \mathrm{nm}$ and a known attenuation $\alpha$ of 0.796 . Fig. 2. gives the result obtained. The presence of ripples might come from residual amplitude modulation into the phase modulator even if a polarization controller is employed.

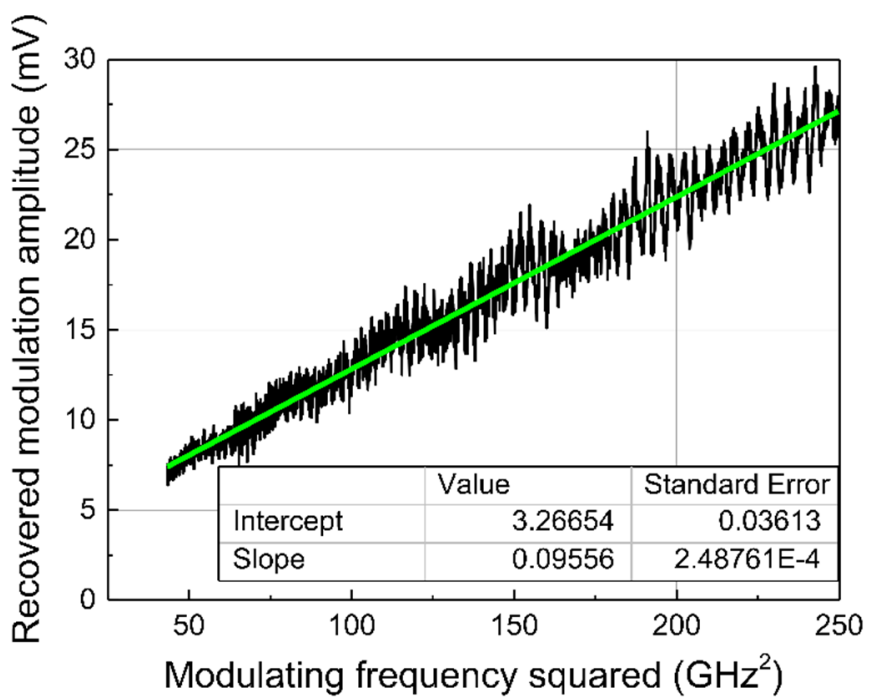

Fig. 3: Extracted linear portion of the recovered modulation amplitude and the corresponding linear fit in green.

Anyway, it can be clearly seen that this amplitude evolves linearly between $6.5 \mathrm{GHz}$ and $15.8 \mathrm{GHz}$. Thus, extracting this portion and performing a linear fit yields a value of $\kappa$ of $4.55 \times 10^{-23} \mathrm{~V} . \mathrm{nm} / \mathrm{ps} / \mathrm{Hz}^{2}$. Fig. 3 shows the result of this calibration. The standard error introduced by the linear fitting leads to an error in the determination of $\kappa$ of $1.18 \times 10^{-2}$. This will corresponds to an error of $0.01 \mathrm{ps} / \mathrm{nm}$ only in the calculation of CD using Eq. (10).

\section{B. CD measurement of short optical fibers}

Once the calibration performed, the total CD of three optical fibers with lengths of $288 \mathrm{~m}, 413 \mathrm{~m}$ and $2 \mathrm{~km}$ has been measured. The evolution of the recovered modulation amplitude for these fibers is plotted in Fig. 4.

By measuring the slope of the linear part of the curves above as well as the attenuation introduced by each fiber, we were able to extract their CD using Eq. (10). The results are reported in table 1.

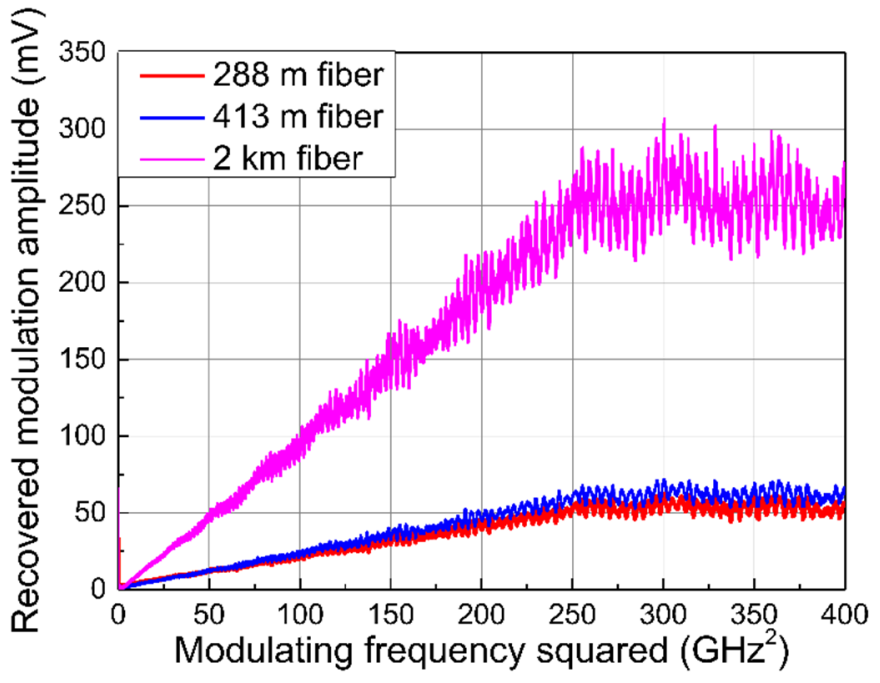

Fig. 4: Evolution of the recovered modulation amplitude for short optical fibers of different length.

\begin{tabular}{|c|c|c|}
\hline DUT & $\begin{array}{c}\mathrm{p}_{\text {measured }} \\
\left(\mathrm{V} / \mathrm{Hz}^{2}\right)\end{array}$ & $\mathrm{D}_{\text {tot }}(\mathrm{ps} / \mathrm{nm})$ \\
\hline $288 \mathrm{~m}$ fiber & $19.6 \times 10^{-23}$ & 4.66 \\
\hline $413 \mathrm{~m}$ fiber & $23.4 \times 10^{-23}$ & 6.46 \\
\hline $2 \mathrm{~km}$ fiber & $96.4 \times 10^{-23}$ & 29.55 \\
\hline \multicolumn{2}{|c|}{ Table 1: Measured total CD of three short optical fibers. }
\end{tabular}

\section{APPLICATION TO THE OPTIMIZATION OF A COEO}

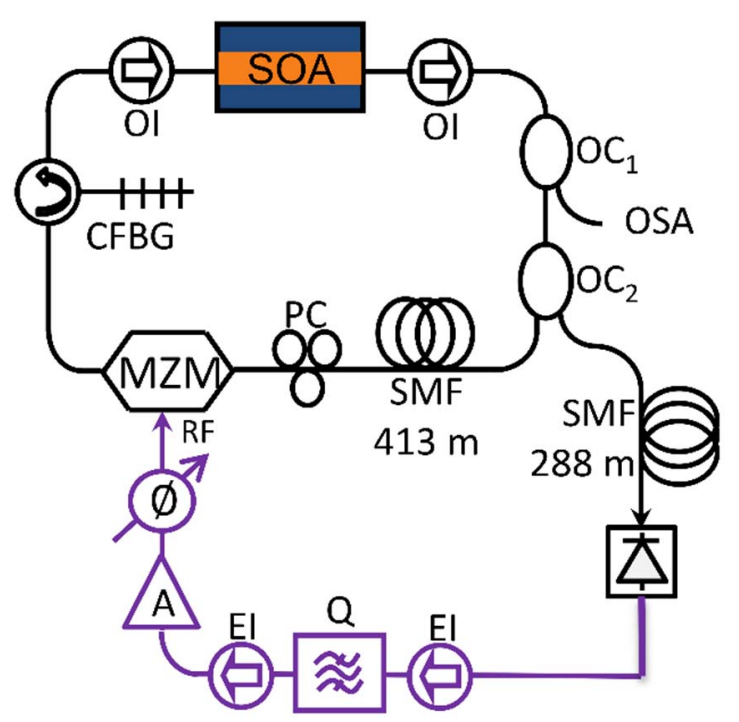

Fig. 5: COEO experimental setup, including the fiber spools and the chirped Bragg filter (CFBG) which have been combined to get a close to zero dispersion in the optical cavity.

The coupled optoelectronic oscillator (Fig. 5) is an efficient way to generate pure microwave signals and optical combs. However, the microwave phase noise performances of the 
COEO are strongly dependent on to the optical pulse temporal width. Indeed, the shorter this width the lower the pulse jitter and, consequently, the lower the phase noise [3]. Thus, in order to generate ultra-high frequency and spectrally pure signals at harmonic frequencies of the COEO fundamental signal, the cavity dispersion of this latter must be carefully optimized. With the approach in Fig. 5 we have demonstrated recently a $90 \mathrm{GHz}$ signal generator (harmonic mixing from a $30 \mathrm{GHz} \mathrm{COEO}$ ) with an ultra-low phase noise level of $-104 \mathrm{dBc} / \mathrm{Hz}$ at $1 \mathrm{kHz}$ offset frequency [7]. The results are displayed on Fig. 6.

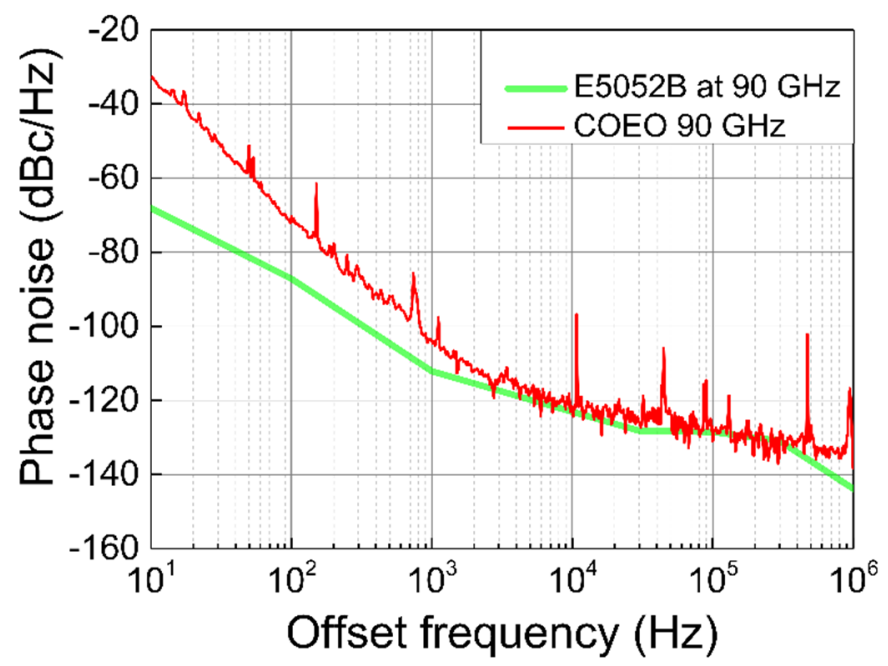

Fig. 6: Phase noise measurements at $90 \mathrm{GHz}$ with a cross correlation technique (correlation factor of 300). Green: phase noise floor of the signal source analyzer at $90 \mathrm{GHz}$.

\section{CONCLUSION}

We have demonstrated a simple method to measure the chromatic dispersion of optical fibers with length in the range of one hundred meters. The resolution of this system is better than $1 \mathrm{ps} / \mathrm{nm}$. We have taken advantage of these results to compensate the chromatic dispersion in an optoelectronic oscillator using a chirped fiber Bragg filter. This oscillator produces high spectral purity reference signals in the millimeterwave range.

\section{ACKNOWLEDGMENTS}

The research on the COEO is supported by French MoD, Délégation Générale à l'Armement.

\section{REFERENCES}

[1] Meslener, G., 1984. Chromatic dispersion induced distortion of modulated monochromatic light employing direct detection. IEEE Journal of Quantum Electronics, 20(10), pp.1208-1216.

[2] Han, X., Zhang, M., Li, S., Shi, N., Gu, Y. and Zhao, M., 2013. A new method for fiber chromatic dispersion measurement with microwave interference effect. Optical Fiber Technology, 19(4), pp.319-324.

[3] Matsko, A.B., Eliyahu, D. and Maleki, L., 2013. Theory of coupled optoelectronic microwave oscillator II: phase noise. JOSA B, 30(12), pp.3316-3323.
[4] Lelièvre, O., Crozatier, V., Baili, G., Nouchi, P., Dolfi, D., Morvan, L., Goldfarb, F., Bretenaker, F. and Llopis, O., 2017, July. In-situ dispersion measurement of a coupled optoelectronic oscillator. In Frequency and Time Forum and IEEE International Frequency Control Symposium (EFTF/IFC), 2017 Joint Conference of the European (pp. 190-192). IEEE.

[5] Yamamoto, T., Kurokawa, K., Tajima, K. and Kurashima, T., 2009, March. Simple and precise chromatic dispersion measurement using sinusoidally phase-modulated $\mathrm{CW}$ light. In Optical Fiber Communication-incudes post deadline papers, 2009. OFC 2009. Conference on (pp. 1-3). IEEE.

[6] Y. Yorozu, M. Hirano, K. Oka, and Y. Tagawa, "Electron spectroscopy studies on magneto-optical media and plastic substrate interface," IEEE

[7] Aliou Ly, Vincent Auroux, Ramin Khayatzadeh, Napoleon Gutierrez, Arnaud Fernandez, Olivier Llopis, "Highly spectrally pure $90 \mathrm{GHz}$ signal synthesis using a coupled optoelectronic oscillator," to be published in IEEE Photonics Technology Letters. 